\title{
Wells Syndrome Successfully Treated with Tripterygium Glycosides
}

Jiejie Lu

Weiwei Wu

Ming Zhang

Ping Wang

Mu Niu

Xianxu Yang

Department of Dermatology, The Fifth People's Hospital of Hainan Province, Branch of National Clinical Research Center for Skin and Immune Disease, Haikou, Hainan, People's Republic of China
Correspondence: Mu Niu; Xianxu Yang Department of Dermatology, The Fifth People's Hospital of Hainan Province, Branch of National Clinical Research Center for Skin and Immune Disease, No. 33, Southern Road of Longkun, Qiongshan District, Haikou, 570206, Hainan, People's Republic of China $\mathrm{Tel}+86-18389485160 ;+86-13876300372$ Fax +86-898-66729550; +86-898-66729550 Email 382219948@qq.com; |3876300372@|39.com

\begin{abstract}
Wells syndrome (WS), also known as eosinophilic cellulitis, is a rare inflammatory dermatosis of unknown etiology that typically presents with pruritic cellulitis-like plaques. The first line treatment options for WS are topical or systemic corticosteroids, however, the development of side effects of systemic corticosteroids usually led to a switch to the second line therapy. Here, we reported a rare case of facial Wells syndrome misdiagnosed with bacterial cellulitis. A 26-year-old female presented with a one-week history of erythematous, edematous and blushing plaques partially covered by bullae. A skin biopsy revealed diffuse infiltration of eosinophils in the entire dermis and "flame figures" compatible with WS. Initially, the patient was successfully treated with methylprednisolone. However, three month later, the disease relapsed. Because of weight gain and centripetal obesity, the patient refused to oral administration of methylprednisolone. Traditional Chinese Medicine tripterygium glycosides (TG) $60 \mathrm{mg} /$ day was prescribed and the lesions completely resolved after 4 weeks without any recurrence. Our case suggests that tripterygium glycosides may be a safe and effective treatment option for Wells syndrome.
\end{abstract}

Keywords: Wells syndrome, tripterygium glycosides, facial cellulitis

\section{Introduction}

Wells syndrome (WS; George Wells, 1971), also known as eosinophilic cellulitis, is a rare inflammatory dermatosis of unknown etiology. ${ }^{1}$ There are numerous treatment regiments available for WS, including general or local corticosteroids, immunomodulatory or immunosuppressive therapies. ${ }^{2}$ Although general corticosteroids was recommended as the reference and mainstay regiment, potential adverse effects of corticosteroids and relapse after withdrawal often limit its clinical application. Chinese herbal medicine was often used as an useful complementary and alternative therapy to corticosteroids therapy in China. Tripterygium wilfordii and its preparations are extracts of Chinese herbal medicine plant Tripterygium wilfordii Hook. f., which mainly contain triptonide, tripdiolide, and triptolide. ${ }^{3}$ Tripterygium glycosides (TG) mainly comprises of triptolides, which has been proved to have anti-inflammatory and immunosuppressive effects. ${ }^{3}$ So, TG has been widely used in the long-term therapy of inflammatory, autoimmune diseases or vasculitis in China, such as psoriasis, rheumatoid arthritis, systemic lupus erythematosus and Behcet's disease. ${ }^{4,5}$ Here, we report a rare case of facial WS that masqueraded as bacterial cellulitis, which successfully treated with tripterygium glycosides.

\section{Case Presentation}

A 26-year-old female presented with a one-week history of pruritic, painful, facial erythematous edema covered by vesicles and yellowish crusts. Facial bacterial 
cellulitis was originally diagnosed by the local general practitioner, and oral antibiotic (cephalexin) was prescribed for 5 days, which did not work. No significant trigger factors, including drugs, arthropod bites or trauma, could be identified. She did not have any systemic symptoms. Clinical examination revealed edematous, erythematous plaques, vesicles and crusts on the face (Figure 1A). The blood routine test revealed a mild peripheral eosinophilia of $0.65 \times 10^{9}$ cells $/ \mathrm{L}\left(0.04 \sim 0.4 \times 10^{9}\right.$ cells $\left./ \mathrm{L}\right)$. The following laboratory studies were within normal limits or negative: routine tests of urine and stool, bacterial cultures, hepatic and renal function tests, blood sugar, C-reactive protein, erythrocyte sedimentation rate, rheumatoid factor, antinuclear antibodies and extractable nuclear antigen. Histological findings presented as a dense dermal perivascular and interstitial lymphocytic infiltrate, some histiocytes and multinucleate giant cells, associated with many eosinophils and "flame figures" compatible with WS (Figure 1C and 1D). The patient was treated with intravenous methylprednisolone $(60 \mathrm{mg} /$ day $)$, resulting in a dramatic improvement in the skin lesions and blood eosinophilia within $48 \mathrm{~h}$. Consequently oral methylprednisolone was tapered and discontinued after 8 weeks. However, three month later, the disease relapsed. Because of weight gain and centripetal obesity, the patient refused to oral administration of methylprednisolone. Traditional Chinese Medicine tripterygium glycosides (TG) $60 \mathrm{mg} /$ day was prescribed and the lesions completely resolved after 4 weeks. Since then, she no longer took any medication and experienced no recurrence of disease during one year of follow-up (Figure 1B).

\section{Discussion}

Weins et al. proposed the revised diagnostic criteria for Wells syndrome based on case series, which requires at least 2 major criteria and at least 1 minor criteria: Major criteria, (a) Typical clinical features; (b) Chronic relapsing course; (c) Exclusion of systemic disease; (d) Histology: eosinophilic infiltrates, no signs of vasculitis; Minor criteria: (a) Histology with'flame figures'; (b) Histology with
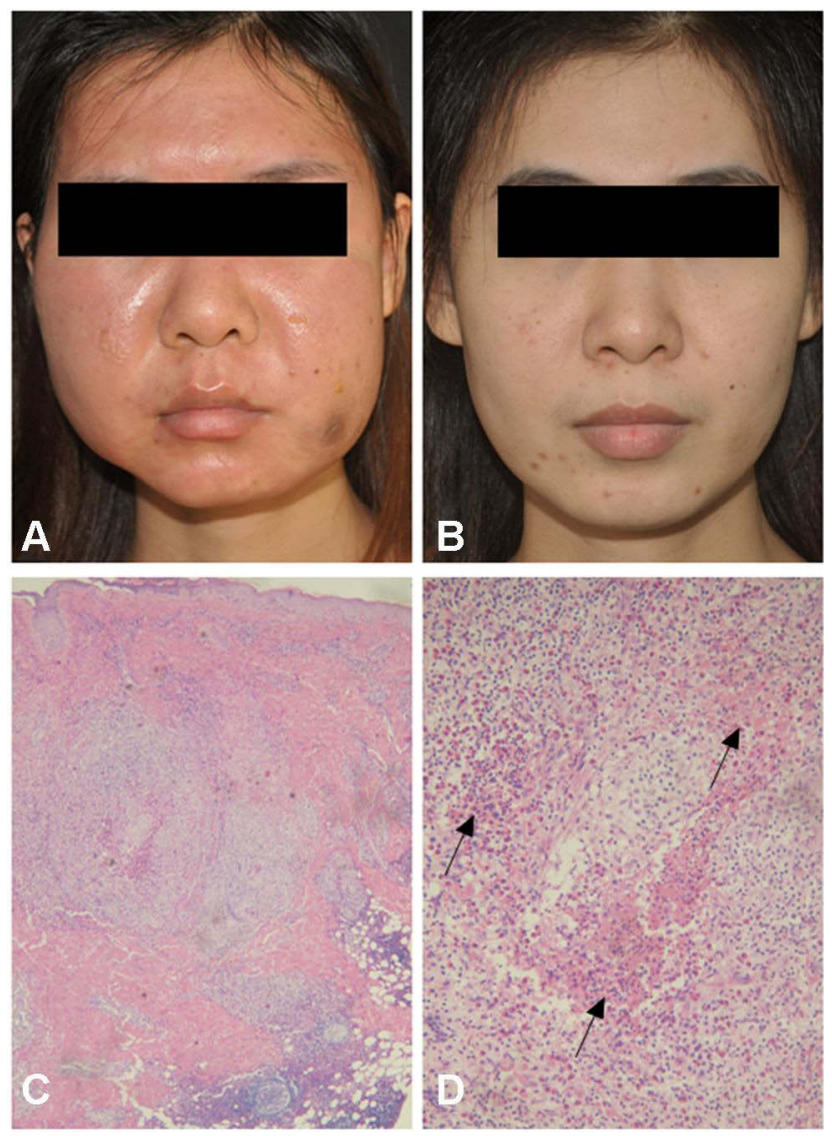

Figure I (A) Facial edematous, erythematous plaques partially covered by vesicles and yellowish crusts. (B) The lesions completely resolved. (C) Dense eosinophilic infiltration in the dermis (HE, 40x). (D) Higher magnification with numerous eosinophils and 'flame figures' (HE, 200x). Degranulated eosinophils forming flame figures (black arrows). 
granulomatous changes; (c) Peripheral eosinophilia up to $1500 / \mu \mathrm{L}$; (d) Detectable triggering factor. ${ }^{6}$ Depending on history and clinicopathological correlation, the patient was diagnosed as Wells syndrome. As TG is widely used in inflammatory dermatosis, we prescribed TG to the patient while the disease relapsed, which led to completely remission without any obvious side effects.

\section{Conclusions}

In summary, we present a patient with Wells syndrome successfully treated with TG. Despite being limited, our clinical experience supports tripterygium glycosides may be one of the effective treatment alternatives in patients who are dependent on or experience side effects with corticosteroids.

\section{Ethics Statement}

The publications of images were included in the patient's consent for publication of the case. The Hospital Ethics Committees of the Fifth People's Hospital of Hainan Province approved to publish the case details.

\section{Consent Statement}

Informed consent was provided by the patient for publication of the case.

\section{Disclosure}

The authors have no conflicts of interest to declare.

\section{References}

1. Wells GC, Smith NP. Eosinophilic cellulitis. Br J Dermatol. 1979;100 (1):101-109. doi:10.1111/j.1365-2133.1979.tb03574.x

2. Räßler F, Lukács J, Elsner P. Treatment of eosinophilic cellulitis (Wells syndrome) - a systematic review. J Eur Acad Dermatol Venereol. 2016;30(9):1465-1479. doi:10.1111/jdv.13706

3. Qiu D, Kao PN. Immunosuppressive and anti-inflammatory mechanisms of triptolide, the principal active diterpenoid from the Chinese medicinal herb Tripterygium wilfordii Hook. Drugs $R$ D. 2003;4 (1):1-18. doi:10.2165/00126839-200304010-00001

4. Han R, Rostami-Yazdi M, Gerdes S, Mrowietz U. Triptolide in the treatment of psoriasis and other immune-mediated inflammatory diseases. Br J Clin Pharmacol. 2012;74(3):424-436. doi:10.1111/ j.1365-2125.2012.04221.x

5. Song CY, Xu YG, Lu YQ. Use of Tripterygium wilfordii Hook F for immune-mediated inflammatory diseases: progress and future prospects. J Zhejiang Univ Sci B. 2020;21(4):280-290. doi:10.1631/ jzus.B1900607

6. Weins $\mathrm{AB}$, Biedermann $\mathrm{T}$, Weiss $\mathrm{T}$, Weiss JM. Wells syndrome. J Dtsch Dermatol Ges. 2016;14(10):989-993. doi:10.1111/ddg.13132

\section{Publish your work in this journal}

Clinical, Cosmetic and Investigational Dermatology is an international, peer-reviewed, open access, online journal that focuses on the latest clinical and experimental research in all aspects of skin disease and cosmetic interventions. This journal is indexed on CAS.
The manuscript management system is completely online and includes a very quick and fair peer-review system, which is all easy to use. Visit http://www.dovepress.com/testimonials.php to read real quotes from published authors. 